\title{
Colossal grain growth yields single-crystal metal foils by contact-free annealing
}

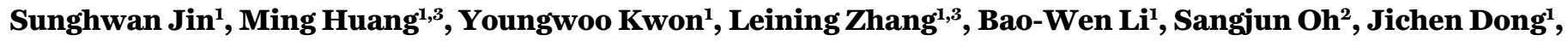 \\ Da Luo', Mandakini Biswal', Benjamin V. Cunning', Pavel V. Bakharev', Inyong Moon ${ }^{4}$, Won Jong Yoo ${ }^{4}$, Dulce \\ C. Camacho-Mojica', Yong-Jin Kim ${ }^{1}$, Sun Hwa Lee', Bin Wang', Won Kyung Seong', Manav Saxena ${ }^{1}$, Feng \\ Ding ${ }^{1,3}$, Hyung-Joon Shin ${ }^{1,3 *}$, Rodney S. Ruoff ${ }^{1,2,3 *}$
}

${ }^{1}$ Center for Multidimensional Carbon Materials (CMCM), Institute for Basic Science (IBS), Ulsan 44919, Republic of Korea. ${ }^{2}$ Department of Chemistry, Ulsan National Institute of Science and Technology (UNIST), Ulsan 44919, Republic of Korea. ${ }^{3}$ School of Materials Science and Engineering, Ulsan National Institute of Science and Technology (UNIST), Ulsan 44919, Republic of Korea. ${ }^{4}$ SKKU Advanced Institute of Nano-Technology (SAINT), Sungkyunkwan University, Suwon, Gyeonggi-do 16419, Republic of Korea.

*Corresponding author. E-mail: shinhj@unist.ac.kr (H.-J.S.); ruofflab@gmail.com or rsruoff@ibs.re.kr (R.S.R.)

Single-crystal metals have unique properties due to the absence of grain boundaries and strong anisotropy. Commercial single-crystal metals are usually synthesized by bulk crystal growth or by deposition of thin films onto substrates, and they are expensive and small. We prepare extremely large single-crystal metal foils by 'contact-free annealing' from commercial polycrystalline foils. The colossal grain growth (up to $32 \mathrm{~cm}^{2}$ ) is achieved by minimizing contact stresses, resulting in a preferred in-plane and out-of-plane crystal orientation, and is driven by surface energy minimization during the rotation of the crystal lattice followed by 'consumption' of neighboring grains. Industrial scale production of singlecrystal metal foils is possible from this discovery.

Polycrystalline metals have numerous grain boundaries (GBs) that affect their electrical and mechanical properties, whereas single-crystal metals have no GBs and show different properties. For example, single-crystal $\mathrm{Cu}$ has a lower electrical resistivity than polycrystalline $\mathrm{Cu}$ due to the elimination of electron scattering at GBs $(1,2)$, and singlecrystal superalloys have a high resistance to creep (3), which can be driven by the sliding of GBs. The growth of graphene on catalytic single-crystal metal substrates by chemical vapor deposition (CVD) has attracted great attention because certain planes have a small lattice mismatch with graphene ( $\mathrm{Cu}(111): \sim 3-4 \%, \mathrm{Ni}(111): \sim 1 \%, \mathrm{Co}(0001): \sim 2 \%)(4,5)$, and can be used as substrates for the heteroepitaxial growth of singlecrystal graphene without GBs (6-10). The epitaxial growth of large scale hexagonal boron nitride, diamond, and tetrahedrally bonded $\mathrm{BN}$ that have a small lattice mismatch with a single-crystal metal has also increased interest in and demand for large area single-crystal metal substrates.

The traditional methods for synthesizing single-crystal metals are by bulk crystal growth (the Czochralski or Bridgman methods). Single-crystal thin metal films can also be deposited on top of single-crystal inorganic substrates $(8,11$ 14). These methods lead to small and expensive single-crystal metals. An alternative strategy is to eliminate GBs in polycrystalline solids by grain growth during annealing. Grain growth produces single-crystal alloy metal sheets but only for the $\mathrm{Cu}-\mathrm{Al}-\mathrm{Mn}$ alloy $(15,16)$. Annealing of polycrystalline $\mathrm{Cu}$ foils yields $\mathrm{Cu}(111)$ oriented foils $(9,10)$, but the mechanism for this transformation remains unclear. We prepared $\mathrm{Cu}(111), \mathrm{Ni}(111), \mathrm{Co}(0001), \mathrm{Pt}(111)$ and $\mathrm{Pd}(111)$ single-crystal foils with a large grain size up to $32 \mathrm{~cm}^{2}$ with "contact-free annealing" (CFA). CFA has advantages as it does not require single-crystal seeds or templates that limit the maximum crystal size, and commercial polycrystalline metal foils are readily converted into single crystals. We heat the foils near their melting temperatures and typically under a hydrogen atmosphere and find an unusual conversion from the polycrystalline grains to a specific orientation in both the in-plane and normal directions. This specific orientation likely arises by crystal lattice rotation accompanied by the removal of stacking faults resulting in a lowered surface energy.

\section{Single-crystal metal foils produced by contact-free annealing}

Grooves typically develop along the GBs on the surface of metals during annealing due to equilibrium between the energy of the free surface and the GBs; such 'thermal grooves' can be observed with the naked eye when the size of individual grains reaches the cm-scale (17-19). We annealed commercial polycrystalline $\mathrm{Cu}$ foils near their melting points with CFA ((20), Fig. 1, A and B). Thermal grooves are typically not present on the surface of the annealed $\mathrm{Cu}$ foil after CFA (Fig. 1C), reflecting an absence of GBs in the entire foil (about 2 $\mathrm{cm} \times 8 \mathrm{~cm}$ in size).

We studied the crystallographic orientations of the annealed $\mathrm{Cu}$ foils by X-ray diffraction (XRD) and electron 
backscatter diffraction (EBSD). We found Bragg reflections from the (111) and (222) crystal planes (Fig. 1D). The inverse pole figure (IPF) color maps from EBSD show only the (111) plane of $\mathrm{Cu}$ (blue color, Fig. 1E), consistent with the XRD data. The entire region also has the same crystallographic orientation in the in-plane direction $(<112>$, the rolling direction), as shown in the pole figures of the (001) poles (Fig. 1F). We found a very small misorientation (below $1^{\circ}$ ) in all kernel average misorientation (KAM) maps, which measure the local average misorientation between a measured point and its neighbors (21) (Fig. 1H). Our measurements show the $\mathrm{Cu}$ foil was converted to a $\{111\}<112>$ single crystal over an area of 2 $\mathrm{cm}$ by $8 \mathrm{~cm}$. In contrast, the grain sizes in thin metal films have a maximum of a few millimeters (22-29), by abnormal grain growth. CFA reproducibly yields (111) single-crystal foils up to $32 \mathrm{~cm}^{2}$ (fig. S1). This colossal grain growth dramatically improves the size of single-crystal foils.

The close-packed $\{111\}$ crystallographic plane of $\mathrm{Cu}$ has the lowest surface energy of all the planes as is well known for face-centered cubic (FCC) structure (30-32). If the surface energy is the major driving force for grain growth in polycrystalline FCC metals they should spontaneously transform into grains with a $\{111\}$ surface due to surface energy minimization (22-24). However, this may not be the case if other energy terms such as strain energy caused by thermal stress are large compared to the surface energy (24). We found that suspending the metal foil eliminates or at least minimizes deformation from thermal stress that arises due to interfacial contact ((20), figs. S2 to S4). This allowed us to reproducibly obtain single grain Ni foils with a \{111\} normal and Co foils with a $\{0001\}$ normal over large areas with the same strategy as for $\mathrm{Cu}((20)$, figs. $\mathrm{S} 5$ to $\mathrm{S} 7)$.

We used resistive heating to convert polycrystalline Pt to single crystal (Fig. 2A) due to the high melting temperature (2041 K). We first attached the Pt foil to two electrodes to pass current, and the Pt foil deforms in the middle from thermal expansion during annealing. This results in GBs near, and in, this deformed region (Fig. 2, B to D). To avoid this we attached the bottom electrode to a movable stage whose position was manually adjusted to keep the foil "flat" during expansion and contraction. We achieved a large area singlecrystal Pt foil region with a $\{111\}$ surface, although the regions near the water-cooled electrode attachment did not convert (Fig. 2, E to I). As long as mechanical deformation is minimized during annealing (fig. S8), we expect this method to work for other foils (it did for Pd, (20) and fig. S9).

\section{Colossal growth of grains with a $\{111\}<112>$ orientation}

We investigated the microstructural and texture evolution by varying annealing time (at $1323 \mathrm{~K}$ ) in order to understand the colossal grain growth. We analyzed the texture of each $\mathrm{Cu}$ foil using the orientation distribution function (ODF) from the
EBSD data (Fig. 3A). The as-received $\mathrm{Cu}$ foil showed mostly elongated grains along the rolling direction, and strong $\{112\}<111>$ and $\{110\}<112>$ texture (Fig. 3B), as expected for a cold-rolled $\mathrm{Cu}$ foil (33). The elongated grains recrystallized to larger polygonal grains after 1 hour of annealing. In addition, small grains with a $\{111\}<112>$ texture $(\{111\}<112>$ grains $)$ started to appear among the $\{112\}$ and $\{110\}$ grains (Fig. 3C). We found that not all $\{111\}$ grains grew to $\mathrm{cm}$-scale. Only a limited number of $\{111\}$ grains located near the edge of the foils grew abnormally, likely due to the energy stored near the foil edge resulting from cutting or handling. Our observations strongly suggest that the stored energy at the edges of the foil accelerates the selective growth of the $\{111\}$ grain(s). These abnormal $\{111\}$ grain(s) grow extremely fast (10s of $\mathrm{mm} / \mathrm{s})$ by consuming the surrounding grains ((20), figs. S10 to S12). This is similar to abnormal grain growth driven by the critical strain annealing process, which promotes the growth of a limited number of grain(s) (34). After 2 hours, we observed two $\mathrm{cm}$-scale grains, separated by numerous $\mu \mathrm{m}$ scale small grains with various orientations. Both of these large grains had a $\{111\}<112>$ texture (Fig. 3D). After we annealed the same $\mathrm{Cu}$ foil for an additional 5.5 hours, GBs (excluding the polygranular regions at the edges) were not found by the naked eye. We did however find a subgrain boundary with SEM at the marked region between the two large $\{111\}<112>$ grains in Fig. 3D (Fig. 3E). This region showed a strong $\{111\}<112>$ texture, and there is no clear contrast difference across the subgrain boundary in the IPF map (Fig. $3 \mathrm{~F})$ which showed only a small misorientation, below $1^{\circ}$, across the subgrain boundary (Fig. 3G). Unlike typical abnormal grain growth, the extremely fast growth of particular $\{111\}$ grain(s) allows further growth up to $\mathrm{cm}$-scale. In addition, those grains (or nuclei) that can grow rapidly, which are limited in number throughout the sample, have an identical $<112>$ in-plane orientation and they form single-crystal foil over large area when they grow larger and coalesce.

\section{Origin of the $\{111\}<112>$ orientation in single-crystal FCC metal foils}

Most of our FCC (111) foils (which had initial $\{112\}<111>$ texture) showed identical orientations of both their in-plane and normal directions $(\{111\}<112>)$ (Fig. $4 \mathrm{~A})$. While the preferential growth of grains with a $<111>$ normal direction in FCC metals by surface energy minimization has been reported (22-24), growth of preferred in-plane orientation in FCC metals has been harder to achieve. We studied various factors that we thought could affect the colossal grain growth of $\{111\}<112>$ : initial textures, misorientation angles, thickness, and purity of the metal foil. We found that the initial textures of foils are critical to obtain single-crystal $\{111\}<112>$ foils. If the strongest texture in the initial foil is not $\{112\}<111>$, it results in the different final orientation such as $\{111\}$ fiber and 
$\{100\}<001>((20)$, tables S1 and S2 and figs. S13 to S18).

We also considered the possibility that the dominant $\{112\}<111>$ orientation of our cold-rolled metal foils was responsible for the final $\{111\}<112>$ orientation after CFA. To test whether $\{111\}<112>$ arises from $\{112\}<111>$, we performed CFA on sub-mm scale $\{112\}<111>$ grains. We found these sub$\mathrm{mm}$ scale $\{112\}<111>$ grains in the polygranular region of the annealed $\mathrm{Cu}$ foil that was in contact with the ceramic holder during CFA. We cut this region to have only $\{112\}<111>$ grains at the edge of the $\mathrm{Cu}$ foil sample, and further annealed in the suspended sample configuration at $1323 \mathrm{~K}$ for 12 hours. We found that $\{112\}<111>$ grains converted to $\{111\}<112>$ grains after CFA without substantial change of microstructure (Fig. 4B). Thus, $\{111\}<112>$ grains originate from $\{112\}<111>$ grains.

A contact-free foil is very likely a stress-free foil, thus the driving force for the continuous rotation required for the transformation from a $\{112\}<111>$ grain to a $\{111\}<112>$ grain was not immediately obvious. We know that annealing near the melting point activates many vacancies in the bulk, and the concentration of these vacancies is further increased by the introduction of hydrogen into the metal. Our density functional theory (DFT) calculations show the formation energy of a single vacancy in bulk $\mathrm{Cu}$ is $1.64 \mathrm{eV}$, but it is $1.20 \mathrm{eV}$ and $0.72 \mathrm{eV}$ when the vacancy is passivated by either one or two H atoms, respectively ((20), fig. S22). This large reduction of the vacancy formation energy must result in a substantial increase in the bulk vacancy concentration. Our experiments and simulations show the importance of hydrogen for grain growth, because vacancies in the bulk are critical for grain rotation and when $\mathrm{H}$ atoms diffuse in these metals they strongly stabilize vacancies. As an exceptional case, Pt foil samples showed $\mathrm{cm}$-scale grain growth during CFA both with, but also without, hydrogen. Because Pt has a much higher vacancy concentration than other metals at the annealing temperature used, we found the Pt foil has a largescale grain without hydrogen ((20), table S3 and figs. S19 to S21). We did molecular dynamics (MD) simulations on a model grain possessing a $\{112\}$ surface with $\sim 4.5 \%$ vacancies in the bulk to further understand the relationship between the vacancies and the grain rotation. We found that, after the simulation, the entire grain rotated $2.02^{\circ}$ toward the $\{111\}$ direction which resulted in the removal of one step on the surface and a $\left\{\begin{array}{llll}7 & 7 & 13\end{array}\right\}$ surface. We introduced additional vacancies $(\sim 5.0 \%)$ to this rotated structure, and after relaxation, a further $7.42^{\circ}$ rotation toward the $\{111\}$ direction was observed resulting in the removal of two additional surface

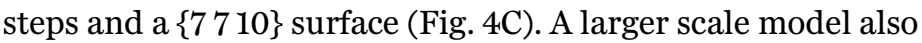
showed a similar result ((20), fig. S23).

Our MD simulations indicate that, with vacancies, the rotation of the $\{112\}$ grain may occur in a stress-free environment. The mechanism we observed has several steps (Fig. 4D): (i) Vacancies first aggregate to form an intrinsic (111) stacking fault (SF) accompanied by Frank partial dislocations (FPDs). (ii) The climbing of the FPD occurs by absorbing more vacancies resulting in SF growth (fig. S24), which eventually spans the entire thickness of the grain, and leads to the disappearance of the FPDs on the surface. (iii) The interaction between the SF and the surface causes a Shockley partial dislocation (SPD) at one side of the SF. This SPD then glides from the surface into the bulk, resulting in continuous shrinkage of the SF area accompanied with rotation of the crystal lattice toward the $\{111\}$ direction. The gliding of the SPD is key in forming the $\{111\}$ surface. Because the gliding of the SPD along the SF results in both the removal of SFs and the forming of a perfect crystal lattice (lower energy state), motion of the SPD is irreversible once SPD initiates from the surface. The gliding of the SPD can initiate in two directions from the surface (Fig. 4E). Initiation in one direction causes the surface step height to increase, while initiation in the other direction results in the removal of the surface step and a flat surface. DFT energy calculations of these states demonstrate that the gliding of the SPD that results in the flat surface is more energetically favorable, and this causes rotation toward the $\{111\}$ orientation. Accordingly, during annealing, the grain surface both flattens and rotates eventually resulting in a $\{111\}$ surface.

These observations show that the grain rotation happens along the 'surface energy minimization pathway' of $\mathrm{Cu}$ from $\{112\}$ to $\{111\}$ (fig. S25) during the migration and elimination of SFs in the $\{112\}$ grain(s), which are the major component $(\{112\}<111>)$ in cold-rolled FCC metal foils. But our experimental results show that the $\{110\}$ grain(s), which are the second major component $(\{110\}<112>)$ in cold-rolled FCC foils, do not rotate during the annealing (fig. S26). The $\{110\}$ orientation is located at a saddle point in the calculated surface energy map (fig. S25), therefore, the driving force for initiating the rotation is zero. The observed rotation from $\{112\}$ to $\{111\}$ is the rotation along the $<110>$ axis, which corresponds to the rotation from the $\{112\}<111>$ orientation to the $\{111\}<112>$ orientation. Once the new $\{111\}<112>$ grain(s) are nucleated by grain rotation, they grow rapidly in the highly textured matrix to minimize the surface energy. This is accelerated by the locally stored energy at the edges. Eventually this transformation forms the large $\{111\}<112>$ grain(s) over the entire foil.

\section{Potential applications of single-crystal metal foils}

These single-crystal metal foils will find many uses in surface science, fundamental catalysis research, and a variety of other applications. As an example of using a single-crystal metal foil as a high quality catalyst, we demonstrated the heteroepitaxial growth of single-crystal monolayer and multilayer graphene islands on a pure $\mathrm{Cu}(111)$ foil and a $\mathrm{Cu} / \mathrm{Ni}(111)$ foil, respectively ((20), figs. S27 to S29). In addition, we 
measured a $7 \%$ reduction in the room temperature resistivity of $\mathrm{Cu}(111)$ foils compared to the as-received $\mathrm{Cu}$ foils (table $\mathrm{S} 4$ and figs. S30 and S31). In another example, we obtained different single orientation surfaces (than (111)) by 'simply' cutting polycrystalline foils and then annealing them (fig. S32).

Contact-free annealing enables preparation of single-crystal metal foils with specific in-plane and out-of-plane crystallographic orientations. The orientation originates from the rotation of the dominant texture component of the as-received foils, likely through dislocation gliding as a result of removal of the stacking faults in the presence of large amounts of vacancies. Our results suggest the possibility of achieving very large single-crystal metal foils from polycrystalline foils for many other metals, in addition to the scaled manufacture of at least $\mathrm{Cu}, \mathrm{Ni}, \mathrm{Co}, \mathrm{Pt}$, and $\mathrm{Pd}$ foils, by a process such as CFA.

\section{REFERENCES AND NOTES}

1. Y. C. Cho, S. Lee, M. Ajmal, W.-K. Kim, C. R. Cho, S.-Y. Jeong, J. H. Park, S. E. Park, S. Park, H.-K. Pak, H. C. Kim, Copper better than silver: Electrical resistivity of the grain-free single-crystal copper wire. Cryst. Growth Des. 10, 2780-2784 (2010). doi:10.1021/cg1003808

2. M. Ajmal, S. Lee, Y. C. Cho, S. J. Kim, S. E. Park, C. R. Cho, S.-Y. Jeong, Fabrication of the best conductor from single-crystal copper and the contribution of grain boundaries to the Debye temperature. CrystEngComm 14, 1463-1467 (2012). doi:10.1039/C1CE06026K

3. F. I. Versnyder, M. E. Shank, The development of columnar grain and single crystal high temperature materials through directional solidification. Mater. Sci. Eng. 6 , 213-247 (1970). doi:10.1016/0025-5416(70)90050-9

4. X. Mi, V. Meunier, N. Koratkar, Y. F. Shi, Facet-insensitive graphene growth on copper. Phys. Rev. B 85, 155436 (2012). doi:10.1103/PhysRevB.85.155436

5. C. M. Seah, S. P. Chai, A. R. Mohamed, Mechanisms of graphene growth by chemical vapour deposition on transition metals. Carbon 70, 1-21 (2014). doi:10.1016/j.carbon.2013.12.073

6. Y. Ogawa, B. Hu, C. M. Orofeo, M. Tsuji, K. Ikeda, S. Mizuno, H. Hibino, H. Ago, Domain structure and boundary in single-layer graphene grown on $\mathrm{Cu}(111)$ and $\mathrm{Cu}(100)$ films. J. Phys. Chem. Lett. 3, 219-226 (2012). doi:10.1021/jz2015555

7. A. Varykhalov, O. Rader, Graphene grown on $\mathrm{Co}(0001)$ films and islands: Electronic structure and its precise magnetization dependence. Phys. Rev. B 80, 035437 (2009). doi:10.1103/PhysRevB.80.035437

8. T. Iwasaki, H. J. Park, M. Konuma, D. S. Lee, J. H. Smet, U. Starke, Long-range ordered single-crystal graphene on high-quality heteroepitaxial $\mathrm{Ni}$ thin films grown on MgO(111). Nano Lett. 11, 79-84 (2011). doi:10.1021/nl102834q Medline

9. V. L. Nguyen, B. G. Shin, D. L. Duong, S. T. Kim, D. Perello, Y. J. Lim, Q. H. Yuan, F. Ding, H. Y. Jeong, H. S. Shin, S. M. Lee, S. H. Chae, Q. A. Vu, S. H. Lee, Y. H. Lee, Seamless stitching of graphene domains on polished copper (111) foil. Adv. Mater. 27, 1376-1382 (2015). doi:10.1002/adma.201404541 Medline

10. L. Brown, E. B. Lochocki, J. Avila, C.-J. Kim, Y. Ogawa, R. W. Havener, D.-K. Kim, E. J. Monkman, D. E. Shai, H. I. Wei, M. P. Levendorf, M. Asensio, K. M. Shen, J. Park, Polycrystalline graphene with single crystalline electronic structure. Nano Lett. 14, 5706-5711 (2014). doi:10.1021/nl502445j Medline

11. B. S. Hu, H. Ago, Y. Ito, K. Kawahara, M. Tsuji, E. Magome, K. Sumitani, N. Mizuta, K. Ikeda, S. Mizuno, Epitaxial growth of large-area single-layer graphene over $\mathrm{Cu}(111) /$ sapphire by atmospheric pressure CVD. Carbon 50, 57-65 (2012). doi:10.1016/j.carbon.2011.08.002

12. K. M. Reddy, A. D. Gledhill, C. H. Chen, J. M. Drexler, N. P. Padture, High quality, transferrable graphene grown on single crystal $\mathrm{Cu}(111)$ thin films on basal-plane sapphire. Appl. Phys. Lett. 98, 113117 (2011). doi:10.1063/1.3569143

13. C. M. Orofeo, H. Hibino, K. Kawahara, Y. Ogawa, M. Tsuji, K. Ikeda, S. Mizuno, H. Ago, o, Influence of Cu metal on the domain structure and carrier mobility in single-layer graphene. Carbon 50, 2189-2196 (2012). doi:10.1016/j.carbon.2012.01.030
14. D. L. Miller, M. W. Keller, J. M. Shaw, A. N. Chiaramonti, R. R. Keller, Epitaxial (111) films of $\mathrm{Cu}$, $\mathrm{Ni}$, and $\mathrm{CuxNiy}$ on alpha- $\mathrm{Al}_{2} \mathrm{O}_{3}(0001)$ for graphene growth by chemical vapor deposition. J. Appl. Phys. 112, 064317 (2012). doi:10.1063/1.4754013

15. T. Omori, T. Kusama, S. Kawata, I. Ohnuma, Y. Sutou, Y. Araki, K. Ishida, R. Kainuma, Abnormal grain growth induced by cyclic heat treatment. Science 341 1500-1502 (2013). doi:10.1126/science.1238017 Medline

16. T. Kusama, T. Omori, T. Saito, S. Kise, T. Tanaka, Y. Araki, R. Kainuma, Ultra-large single crystals by abnormal grain growth. Nat. Commun. 8, 354 (2017) doi:10.1038/s41467-017-00383-0 Medline

17. W. W. Mullins, Theory of thermal grooving. J. Appl. Phys. 28, 333-339 (1957). doi:10.1063/1.1722742

18. W. W. Mullins, The effect of thermal grooving on grain boundary motion. Acta Metall. Mater. 6, 414-427 (1958). doi:10.1016/0001-6160(58)90020-8

19. H. J. Frost, C. V. Thompson, D. T. Walton, Simulation of thin-film grain structures. 1. grain-growth stagnation. Acta Metall. Mater. 38, 1455-1462 (1990). doi:10.1016/0956-7151(90)90114-V

20. Materials, methods and additional data are available as supplementary materials.

21. S. I. Wright, M. M. Nowell, D. P. Field, A review of strain analysis using electron backscatter diffraction. Microsc. Microanal. 17, 316-329 (2011). doi:10.1017/S1431927611000055 Medline

22. R. Carel, C. V. Thompson, H. J. Frost, Computer simulation of strain energy effects vs surface and interface energy effects on grain growth in thin films. Acta Mater 44, 2479-2494 (1996). doi:10.1016/1359-6454(95)00365-7

23. C. C. Wong, H. I. Smith, C. V. Thompson, Surface-energy-driven secondary graingrowth in thin Au films. Appl. Phys. Lett. 48, 335-337 (1986) doi:10.1063/1.96543

24. C. V. Thompson, R. Carel, Stress and grain growth in thin films. J. Mech. Phys. Solids 44, 657-673 (1996). doi:10.1016/0022-5096(96)00022-1

25. M. Seita, C. M. Pecnik, S. Frank, R. Spolenak, Direct evidence for stress-induced texture evolution and grain growth of silver thin films upon thermal treatment and self-ion bombardment. Acta Mater. 58, 6513-6525 (2010). doi:10.1016/j.actamat.2010.08.014

26. D. S. Lee, D.-Y. Park, H.-J. Woo, S.-H. Kim, J. Ha, E. Yoon, Preferred orientation controlled giant grain growth of platinum thin films on $\mathrm{SiO}_{2} / \mathrm{Si}$ substrates. Jpn. J. Appl. Phys. 40, L1-L3 (2001). doi:10.1143/JJAP.40.L1

27. J. Greiser, P. Mullner, E. Arzt, Abnormal growth of "giant" grains in silver thin films. Acta Mater. 49, 1041-1050 (2001). doi:10.1016/S1359-6454(00)00372-4

28. A. Gangulee, F. M. Dheurle, Anomalous large grains in alloyed aluminum thinfilms.1. secondary grain-growth in aluminum-copper films. Thin Solid Films 12, 399-402 (1972). doi:10.1016/0040-6090(72)90103-4

29. J. Greiser, D. Muller, P. Mullner, C. V. Thompson, E. Arzt, Growth of giant grains in silver thin films. Scr. Mater. 41, 709-714 (1999). doi:10.1016/S13596462(99)00205-5

30. G. C. Kallinteris, N. I. Papanicolaou, G. A. Evangelakis, D. A. Papaconstantopoulos, Tight-binding interatomic potentials based on total-energy calculation: Application to noble metals using molecular-dynamics simulation. Phys. Rev. B 55, 2150-2156 (1997). doi:10.1103/PhysRevB.55.2150

31. L. Vitos, A. V. Ruban, H. L. Skriver, J. Kollar, The surface energy of metals. Surf. Sci. 411, 186-202 (1998). doi:10.1016/S0039-6028(98)00363-X

32. Q. Jiang, H. M. Lu, M. Zhao, Modelling of surface energies of elemental crystals. J. Phys. Condens. Matter 16, 521-530 (2004). doi:10.1088/0953-8984/16/4/001

33. S. Suwas, R. K. Ray, Crystallographic texture of materials. Engineering materials and processes (Springer, London, 2014).

34. C. P. Blankenship Jr., W. T. Carter Jr., A. E. Murut, M. F. Henry, Recrystallization and grain-growth in strain gradient samples. Scr. Metall. Mater. 31, 647-652 (1994). doi:10.1016/0956-716X(94)90204-6

35. T. A. Hahn, R. K. Kirby, H. C. Wolfe, M. G. Graham, H. E. Hagy, Thermal Expansion of Platinum from 293 to 1900 K. AlP Conf. Proc. 3, 87-95 (1972). doi:10.1063/1.2948578

36. G. Kresse, J. Hafner, Ab initio molecular dynamics for open-shell transition metals. Phys. Rev. B Condens. Matter 48, 13115-13118 (1993). doi:10.1103/PhysRevB.48.13115 Medline

37. G. Kresse, J. Furthmuller, Efficiency of ab-initio total energy calculations for metals and semiconductors using a plane-wave basis set. Comput. Mater. Sci. 6, 15-50 (1996). doi:10.1016/0927-0256(96)00008-0 
38. J. P. Perdew, K. Burke, M. Ernzerhof, Generalized gradient approximation made simple. Phys. Rev. Lett. 77, 3865-3868 (1996). doi:10.1103/PhysRevLett.77.3865 Medline

39. G. Kresse, D. Joubert, From ultrasoft pseudopotentials to the projector augmented-wave method. Phys. Rev. B 59, 1758-1775 (1999). doi:10.1103/PhysRevB.59.1758

40. S. Plimpton, Fast parallel algorithms for short-range molecular dynamics. J. Comput. Phys. 117, 1-19 (1995). doi:10.1006/jcph.1995.1039

41. X. W. Zhou, H. N. G. Wadley, R. A. Johnson, D. J. Larson, N. Tabat, A. Cerezo, A. K. Petford-Long, G. D. W. Smith, P. H. Clifton, R. L. Martens, T. F. Kelly, Atomic scale structure of sputtered metal multilayers. Acta Mater. 49, 4005-4015 (2001). doi:10.1016/S1359-6454(01)00287-7

42. B. Wang, M. Huang, L. Tao, S. H. Lee, A.-R. Jang, B.-W. Li, H. S. Shin, D. Akinwande, R. S. Ruoff, Support-free transfer of ultrasmooth graphene films facilitated by selfassembled monolayers for electronic devices and patterns. ACS Nano 10, 14041410 (2016). doi:10.1021/acsnano.5b06842 Medline

43. W. Betteridge, Properties of metallic cobalt. Prog. Mater. Sci. 24, 51-142 (1979). doi:10.1016/0079-6425(79)90004-5

44. L. E. Murr, E. V. Esquivel, Observations of common microstructural issues associated with dynamic deformation phenomena: Twins, microbands, grain size effects, shear bands, and dynamic recrystallization. J. Mater. Sci. 39, 1153-1168 (2004). doi:10.1023/B:JMSC.0000013870.09241.c0

45. C. V. Thompson, H. I. Smith, Surface-energy-driven secondary grain growth in ultrathin $(<100 \mathrm{~nm})$ films of silicon. Appl. Phys. Lett. 44, 603-605 (1984). doi:10.1063/1.94842

46. R. Bauer, E. A. Jagle, W. Baumann, E. J. Mittemeijer, Kinetics of the allotropic HCPFCC phase transformation in cobalt. Philos. Mag. 91, 437-457 (2011). doi:10.1080/14786435.2010.525541

47. Y. V. Khlebnikova, D. P. Rodionov, V. A. Sazonova, T. I. Tabatchikova, O. V. Antonova, Y. E. Turkhan, V. A. Kazantsev, Study of the structure of cobalt single crystals during the $\beta \rightarrow \alpha$ transformation. Phys. Met. Metallogr. 103, 609-618 (2007). doi:10.1134/S0031918X07060105

48. F. A. Lewis, The hydrides of palladium and palladium alloys. Platin. Met. Rev. 4 132-137 (1960).

49. M. A. Tschopp, D. L. McDowell, Asymmetric tilt grain boundary structure and energy in copper and aluminium. Philos. Mag. 87, 3871-3892 (2007). doi:10.1080/14786430701455321

50. T. A. Bennett, P. N. Kalu, A. D. Rollett, Strain-induced selective growth in $1.5 \%$ temper-rolled Fe 1\%Si. Microsc. Microanal. 17, 362-367 (2011). doi:10.1017/S1431927611000377 Medline

51. F. J. Humphreys, M. Hatherly, Recrystallization and related annealing phenomena. (Elsevier, Amsterdam; Boston, ed. 2nd, 2004).

52. L. S. Shvindlerman, G. Gottstein, D. A. Molodov, V. G. Sursaeva, "Triple junction motion in metals" in Recrystallization and Grain Growth, G. Gottstein, D.A. Molodov, Eds. (Springer-Verlag, Berlin, 2001), vol. 1 and 2, 177-192.

53. D. Wolf, Structure-energy correlation for grain-boundaries in FCC Metals. 3. symmetrical tilt boundaries. Acta Metall. Mater. 38, 781-790 (1990). doi:10.1016/0956-7151(90)90030-K

54. G. Gottstein, L. S. Shvindlerman, Grain boundary migration in metals: thermodynamics, kinetics, applications. CRC series in materials science and technology (Taylor \& Francis, Boca Raton, ed. 2nd, 2010).

55. E. R. Homer, S. Patala, J. L. Priedeman, Grain boundary plane orientation fundamental zones and structure-property relationships. Sci. Rep. 5, 15476 (2015). doi:10.1038/srep15476 Medline

56. R. E. Reed-Hill, R. Abbaschian, Physical metallurgy principles. PWS-Kent series in engineering (PWS-Kent Pub., Boston, ed. 3rd, 1992).

57. Y. Fukai, Formation of superabundant vacancies in metal hydrides at high temperatures. J. Alloys Compd. 231, 35-40 (1995). doi:10.1016/09258388(95)01834-4

58. V. A. Gol'tsov, D. A. Glyakov, G. I. Zhirov, Effect of dissolved hydrogen on the recrystallization and recovery of mechanical properties of deformed palladium. Phys. Met. Metallogr. 102, 318-323 (2006). doi:10.1134/S0031918X06090122

59. Y. Ateba Betanda, A. L. Helbert, F. Brisset, T. Waeckerle, T. Baudin, Effect of annealing atmosphere on the recrystallized texture and abnormal grain growth of Ni-5\% W alloy sheets. Adv. Eng. Mater. 17, 1568-1572 (2015). doi:10.1002/adem.201500071

60. T. Fujikawa, T. Yoshikawa, T. Ohnishi, T. Sato, Hydrogen promoted copper migration in the high pressure anneal process. Jpn. J. Appl. Phys. 40 (Part 1, No. 4A), 2191-2196 (2001). doi:10.1143/JJAP.40.2191

61. W. R. Wampler, T. Schober, B. Lengeler, Precipitation and trapping of hydrogen in copper. Philos. Mag. 34, 129-141 (1976). doi:10.1080/14786437608228179

62. J. E. Jensen, W. A. Tuttle, H. Brechna, A. G. Prodell, "Brookhaven National Laboratory Selected Cryogenic Data Notebook" (Brookhaven National Laboratory, BNL 10200-R, 1980).

63. K. C. Mills, Recommended values of thermophysical properties for selected commercial alloys. (Woodhead, Cambridge, 2002).

64. J. W. Arblaster, Crystallographic properties of platinum. Platin. Met. Rev. 56, 181189 (2012). doi:10.1595/147106712X646113

65. B. Zhang, W. H. Lee, R. Piner, I. Kholmanov, Y. Wu, H. Li, H. Ji, R. S. Ruoff, Lowtemperature chemical vapor deposition growth of graphene from toluene on electropolished copper foils. ACS Nano 6, 2471-2476 (2012). doi:10.1021/nn204827h Medline

66. G. H. Han, F. Günes, J. J. Bae, E. S. Kim, S. J. Chae, H.-J. Shin, J.-Y. Choi, D. Pribat, $Y$. H. Lee, Influence of copper morphology in forming nucleation seeds for graphene growth. Nano Lett. 11, 4144-4148 (2011). doi:10.1021/nl201980p Medline

67. D. W. Kim, Y. H. Kim, H. S. Jeong, H. T. Jung, Direct visualization of large-area graphene domains and boundaries by optical birefringency. Nat. Nanotechnol. 7 29-34 (2011). doi:10.1038/nnano.2011.198 Medline

68. Y. Wang, Y. Zheng, X. Xu, E. Dubuisson, Q. Bao, J. Lu, K. P. Loh, Electrochemical delamination of CVD-grown graphene film: Toward the recyclable use of copper catalyst. ACS Nano 5, 9927-9933 (2011). doi:10.1021/nn203700w Medline

69. Z. Zhao, Z. Shan, C. Zhang, Q. Li, B. Tian, Z. Huang, W. Lin, X. Chen, H. Ji, W. Zhang, W. Cai, Study on the diffusion mechanism of graphene grown on copper pockets. Small 11, 1418-1422 (2015). doi:10.1002/smll.201402483 Medline

70. M. Huang, M. Biswal, H. J. Park, S. Jin, D. Qu, S. Hong, Z. Zhu, L. Qiu, D. Luo, X. Liu, Z. Yang, Z. Liu, Y. Huang, H. Lim, W. J. Yoo, F. Ding, Y. Wang, Z. Lee, R. S. Ruoff, Highly oriented monolayer graphene grown on a $\mathrm{Cu} / \mathrm{Ni}(111)$ alloy foil. ACS Nano 12, 6117-6127 (2018). doi:10.1021/acsnano.8b02444 Medline

71. D. Chapman, "High conductivity copper for electrical engineering" (Copper Development Association's resource library, vol. 122, 1-32, 2016).

\section{ACKNOWLEDGMENTS}

The authors thank Prof. Peter Thrower for comments on manuscript preparation. Funding: This work was supported by IBS-R019-D1. Author contributions: R.S.R. and S.J. conceived the experiments. S.J., Y.K. and S.O. performed the annealing and polishing of the metal foils, and their characterizations. R.S.R. supervised the project. H.J.S. provided critical insight about the texture of metal foils and their influence. B.L. and M.H. performed graphene growth experiments. B.L., M.H. and B.W. performed graphene transfer and characterization. M.H. and M.B. performed POM characterization of graphene. S.J. and D.L. performed AFM analysis. F.D. provided the model about the rotation of grains. L.Z., J.D. and F.D. performed DFT and MD simulations. I.M. and W.J.Y measured the resistivity of the metal foils. P.V.B. and S.J. performed the residual gas analysis experiment. B.C. and D.C. provided comments on the manuscript. M.S. provided the blueprint of the quartz holder. S.H.L performed DSC measurements. W.K.S prepared the customized equipment for fast annealing. S.J., R.S.R., H.J.S and Y.J.K. wrote the manuscript. All co-authors revised and commented on the manuscript. Competing interests: A patent was filed and has been issued (KR 101878465B), and a PCT application has been published (WO 2018/012864 A1) by the Institute for Basic Science (IBS) and Ulsan National Institute of Science and Technology (UNIST), along with their researchers (R.S.R and S.J.). Data and materials availability: All data are available in the main text or the supplementary materials. 


\section{SUPPLEMENTARY MATERIALS}

www.sciencemag.org/cgi/content/full/science.aao3373/DC1

Materials and Methods

Supplementary Text

Figs. S1 to S32

Tables S1 to S4

References (35-71)

11 July 2017; resubmitted 24 August 2018

Accepted 8 October 2018

Published online 18 October 2018

10.1126/science.aao3373 


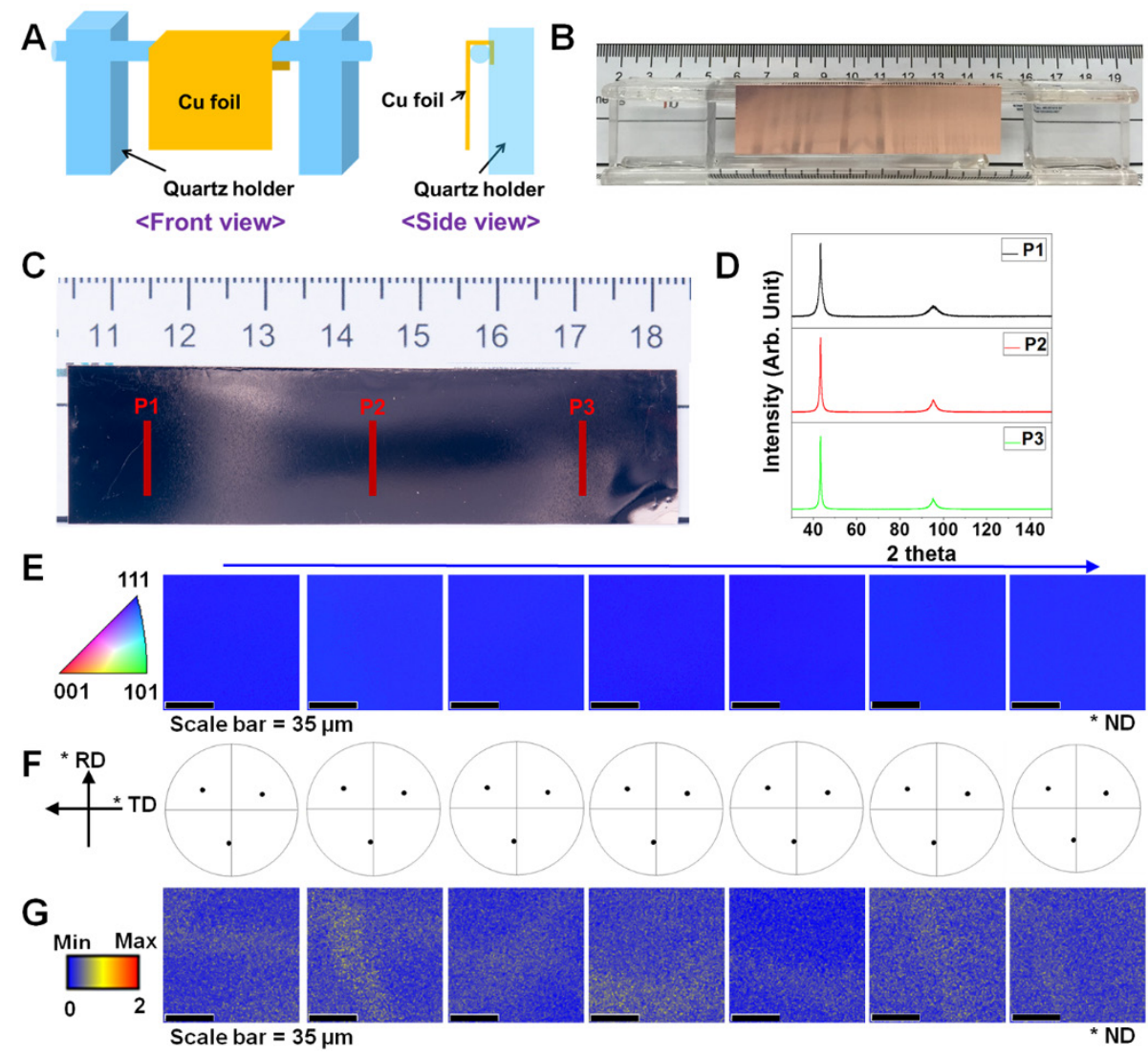

Fig. 1. A single-crystal $\mathrm{Cu}(111)$ foil produced by CFA. (A) Schematic of the quartz holder that the $\mathrm{Cu}$ foil is suspended from, (B) photograph of the configuration shown schematically in (A). (C) Photograph of the annealed single-crystal $\mathrm{Cu}$ foil (the larger numbered lines on the ruler in the photographs are centimeters), (D) The XRD $2 \theta$ scans of the three regions in the annealed single-crystal $\mathrm{Cu}$ foil indicated by P1-P3 in (C) by using $\mathrm{Cu}-\mathrm{K} \alpha$ radiation ( $45 \mathrm{kV}$ and $200 \mathrm{~mA}$ ). The peaks at 43.3 and 95.1 are assigned to Bragg reflections from the (111) and (222) crystallographic planes. (E) EBSD IPF maps in the normal direction. (F) (001) pole figures. (G) KAM maps ( $1^{\text {st }}$ nearest-neighbor kernel) of the single-crystal $\mathrm{Cu}$ foil at various points along the section from P1 to P3 (the distance between adjacent EBSD measurement points was more than $5 \mathrm{~mm})$. $(\mathrm{ND}=$ normal direction, $\mathrm{RD}=$ rolling direction, $\mathrm{TD}=$ transverse direction). 

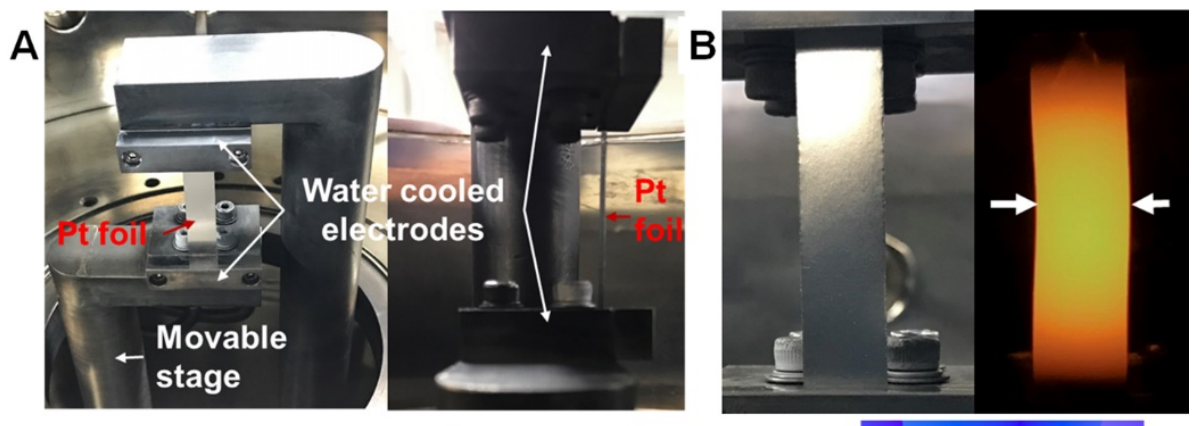

C

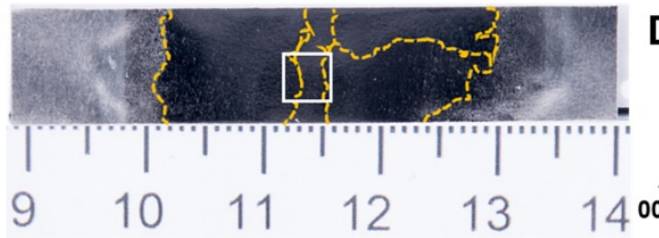

E
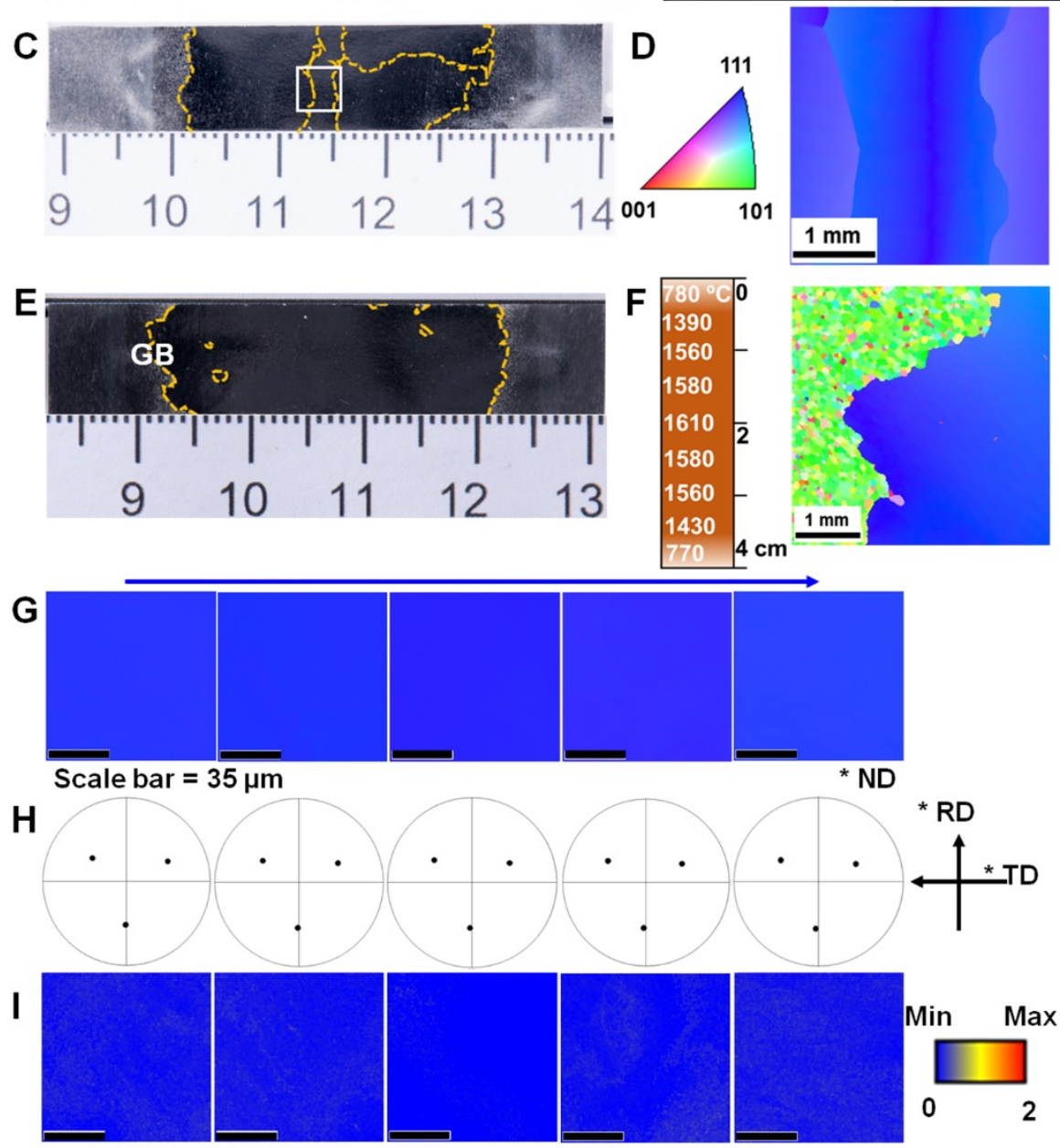

Scale bar $=35 \mu \mathrm{m}$
Fig. 2. A single-crystal $\mathrm{Pt}(111)$ foil region produced by CFA. (A) Photograph of water-cooled electrodes with a Pt foil for resistive heating (left: front view, right: side view). Different from CFA with a ceramic holder, we fixed the foil to two water-cooled electrodes. (B) Photograph of a $\mathrm{Pt}$ foil before heating (left), and then during heating (right) but without stage movement; white arrows indicate bending of the foil. (C) Photograph of the same annealed Pt foil (yellow dashed lines correspond to GBs between large single-grain and polygranular regions or between large grains that have orientations close to (111)). (D) EBSD IPF map of the white square region in $(C)$. (E) Photograph of a single-crystal Pt foil (about $3 \mathrm{~cm}^{2}$ in the singlecrystal region; yellow dashed lines indicate GBs between the singlegrain and polygranular regions) after annealing with appropriate movement of one electrode (stage) to minimize the thermal stress during annealing and cooling. ( $F$ ) Temperature distribution in a Pt foil during annealing (left), and EBSD IPF map of the region marked "GB" in (E). (G) EBSD IPF maps, $(H)$ (001) pole figures, (I) KAM maps (1 (1t $^{\text {st }}$ nearest-neighbor kernel) of the single-crystal Pt foil at various points along its length in the large single grain region (the distance between each EBSD measurement point was more than $5 \mathrm{~mm}$ ). All these foils were annealed at $1883 \mathrm{~K}$ for 12 hours (ND = normal direction, $\mathrm{RD}=$ rolling direction, $\mathrm{TD}=$ transverse direction). 

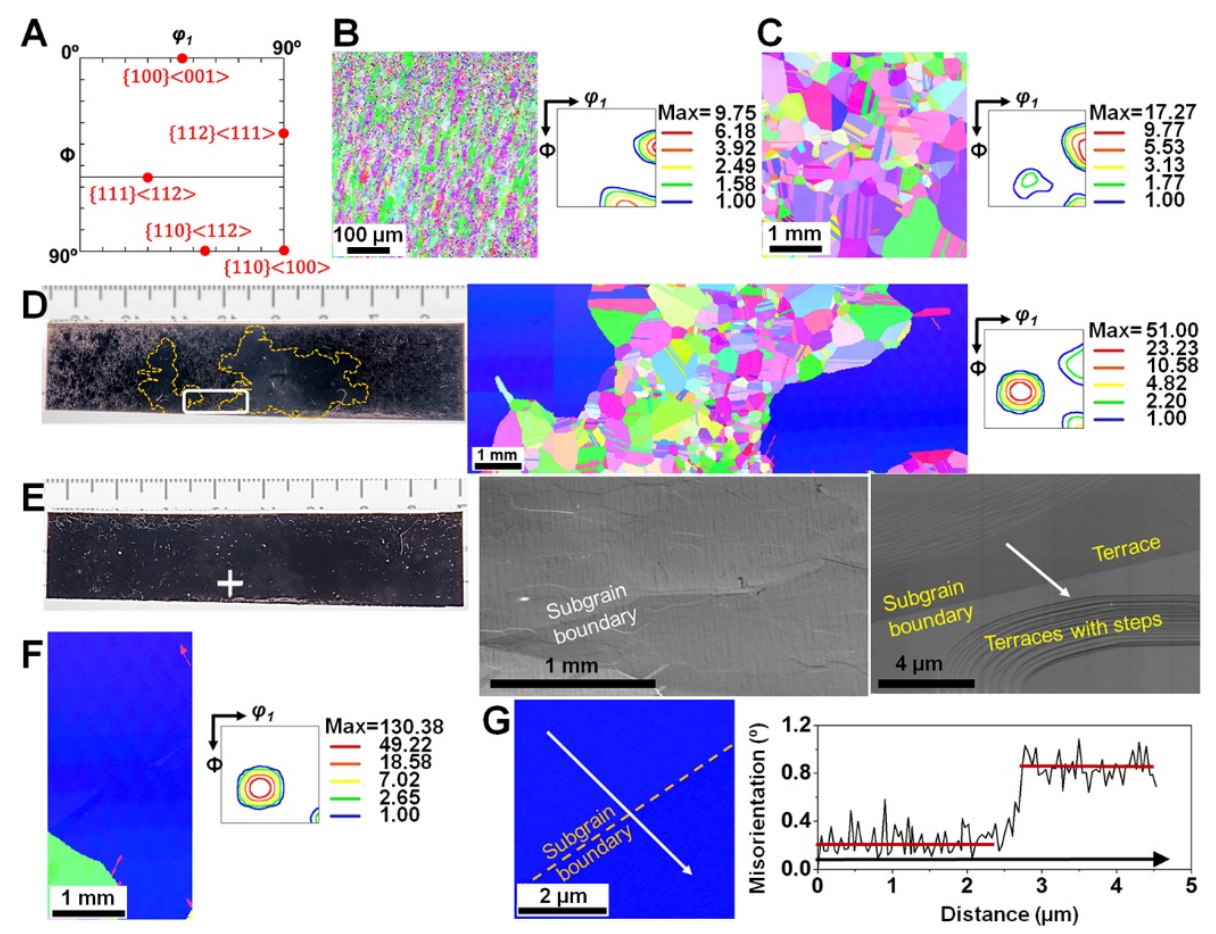

Fig. 3. Texture evolution and grain growth for the large area singlecrystal Cu. (A) ODF section with $\varphi_{2}=45^{\circ}$ indicating the position of the major components of the texture in FCC metals. IPF (left) and ODF (right) maps ( $\left.\Phi, \varphi_{1}=0 \sim 90^{\circ}, \varphi_{2}=45^{\circ}\right)$ of (B) an as-received Cu foil, (C) a Cu foil annealed at 1,323 $\mathrm{K}$ for 1 hour. (D) A Cu foil annealed at 1,323 K for 2 hours: photograph (left, yellow dashed lines correspond to GBs between large single-grain and polygranular regions), and IPF (middle), ODF (right) maps of the region marked with a white rectangle in the photograph. (E) The same Cu foil in (D) after it was annealed for an additional 5.5 hours at $1323 \mathrm{~K}$ : photograph (left), and SEM (middle) and its higher magnification SEM (right) images of the region marked with a white cross in the photograph. (F) IPF (left) and ODF (right) maps at the same region as the lower magnification SEM in (E). (G) IPF (left) maps at the same region as the higher magnification SEM in (E) and the misorientation profile along the white arrow in this higher magnification SEM image and the corresponding IPF map. Note that all SEM images are tilted $70^{\circ}$ because it is necessary to use a sample tilt of $70^{\circ}$ for EBSD observation. 


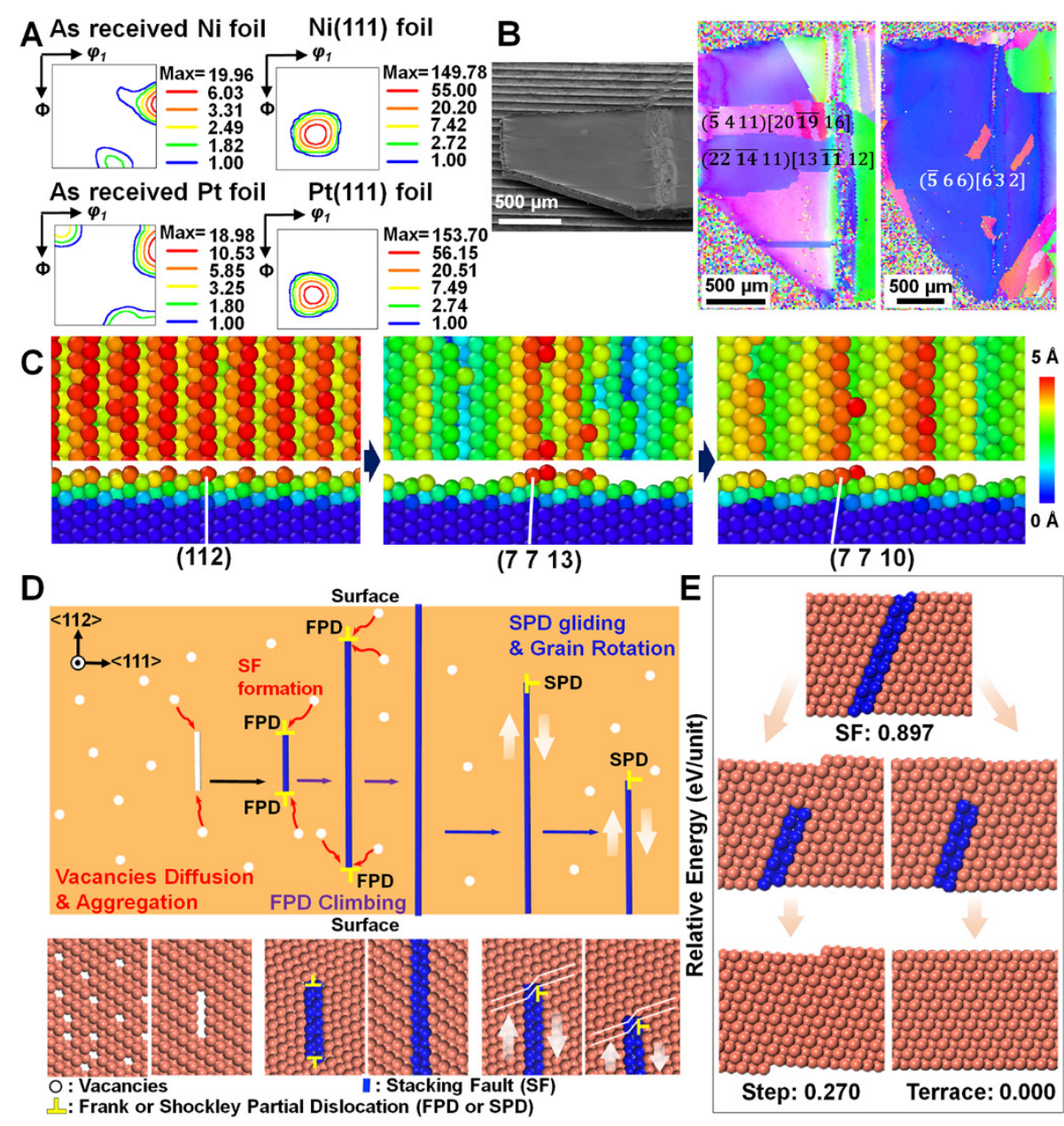

Fig. 4. Transformation from the $\{112\}<111>$ to the $\{111\}<112>$ orientation for the single-crystal FCC foils. (A) ODF section with $\varphi_{2}=45^{\circ}$ of as-received $\mathrm{Ni}$ and Pt foils, and $\mathrm{Ni}(111)$ and $\mathrm{Pt}(111)$ foils $\left(\Phi, \varphi_{1}=0 \sim 90^{\circ}\right)$, (B) SEM image of the isolated $\{112\}<111>$ grain region (left), IPF maps of this region before (middle) and after (right) CFA, (C) Cu grain model viewed in the surface normal (top) and $<110>$ direction (bottom) surface: initial $\{112\}$ grain (left), annealing at $1000 \mathrm{~K}$ for $10 \mathrm{~ns}$ with $4.5 \%$ vacancies (middle), further annealing at the same condition with $5.0 \%$ vacancies (right). The color profile on the right represents the relative height of surface. The slab contains 3,604 atoms with $5.01 \times 2.04 \times 4.40 \mathrm{~nm}^{3}$. (D) Schematics of grain rotation during the removal of SF by dislocation glide (top) and its corresponding MD model (bottom), (E) relative energy of Cu unit cell with 198 atoms. 